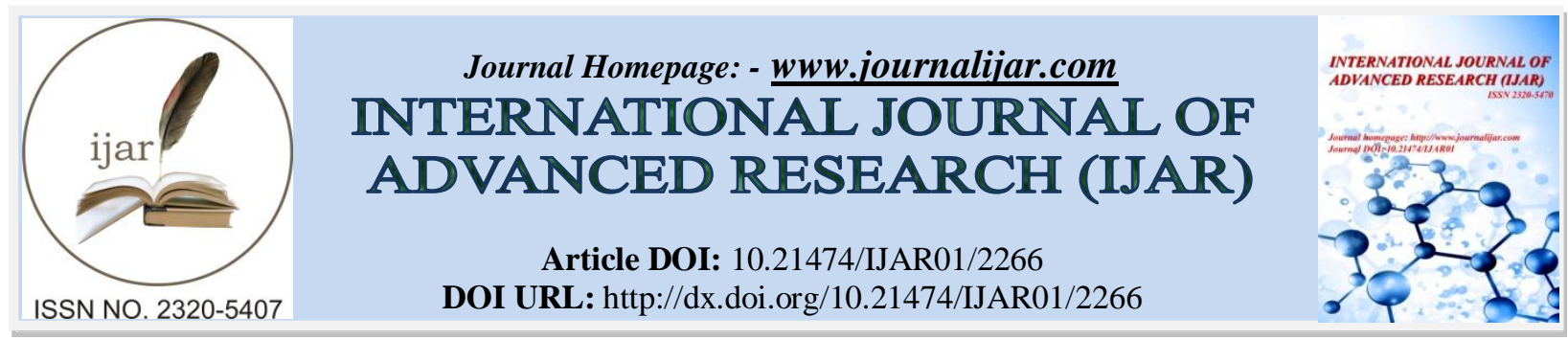

RESEARCH ARTICLE

\title{
IN-VITRO ANTIMICROBIAL STUDY OF BIO-FABRICATED SILVER NANOPARTICLE USING FRUIT EXTRACT OF PHYLLANTHUS EMBLICA.
}

*Amiya kumar Prusty and Nirlipta Behura.

Institute of Pharmacy and Technology, Salipur, Cuttack, Odisha -754 202, India.

\section{Manuscript Info}

Manuscript History

Received: 29 September 2016

Final Accepted: 30 October 2016

Published: November 2016

Key words:-

Silver nanoparticles; fruit extract; SEM analysis; Antimicrobial activity.

\begin{abstract}
Cheap and eco-friendly process for fabrication of silver nanoparticles is an important step in the field of nanotechnology. One of such options is to use easily available biological sources. In the present study use of aqueous fruit extract of Phyllanthus emblica to develop silver nanoparticles by reduction of $\mathrm{Ag}^{+}$was reported. The synthesis process was very fast and silver nanoparticles were formed within minutes of silver ion coming in contact with the fruit extract. UV-visible spectrum of the reaction mixture demonstrated a peak at $454 \mathrm{~nm}$ corresponding to the plasmon absorbance of silver nanoparticles. FTIR analysis of the nanoparticles indicated the presence of proteins, which may be acting as capping agents around the nanoparticles thereby increasing its stability. From scanning electron microscopy (SEM) analysis, the size of the silver nanoparticles was measured and found below $100 \mathrm{~nm}$. Further the antimicrobial activity of synthesized silver nanoparticles showed effective inhibitory activity against tested microorganisms.
\end{abstract}

Copy Right, IJAR, 2016,. All rights reserved.

\section{Introduction:-}

Metal nanoparticles specifically silver nanoparticles have received considerable attention in recent years because of their potential applications in pharmaceutical field, which have a high specific surface area and fraction of surface atoms.

Silver nanoparticles (AgNPs) are synthesized by several physical, chemical and biological method[1]. Such nanoparticles possess unique electrical, optical as well as biological properties and thus applied in catalysis, biosensing, imaging, drug delivery, nanodevice fabrication and also used in medicinal topical ointments to prevent infection against burnand open wounds[2]. Initially nanoparticle synthesis are usually carried out by variousphysical and chemical methods like laser ablation, pyrolysis, lithography, chemical vapour deposition, sol-gel technique and electro deposition, which are very expensive and hazardous[3]. In contrast to this several groups have achieved success in the plant mediated biological synthesis of AgNPs using extracts obtained from Anacardium occidentale, Gloriosasuperba, Hibiscus cannabinus, Malvaparviflora, Ocimumtenuiflorum, Sesbania grandiflora, Mangiferaindica, Prosopisjuliflora and Cocculus hirsutus[4,5,6.7]. In continuation of this efforts for synthesizing AgNPs by green route, a facile, rapid, cheaper and single pot aqueous biosynthesis of AgNPs using the fruit extract of Phyllanthus emblica have been reported here. Phyllanthus emblica is a deciduous tree, commonly known as 'Indian gooseberry' or 'amla; and 'Nelli' in tamil. It belongs to the family Phyllanthaceae, one of the important herbal drugs used in Unani and Ayurvedic systems of medical preparations against a variety conditions such as liver injury, atherosclerosis and diabetes. Earlier studies have demonstrated that it shows potent antimicrobial, 
antioxidant, anti-inflammatory, analgesic and antipyretic, hepatoprotective, antitumor, antiulcerogenic and antidiarrheal. In the present study, we attempted bioinspired nanosynthesis using the aqueous fruit extract of Phyllanthus emblica through various characterization techniques like UV-visible spectroscopy, Scanning Electron Microscopy (SEM), Fourier transform infrared spectroscopy (FTIR). Thus by optimizing the experimental conditions such as silver ion concentration, fruit extract concentration and time, a polydispersed AgNPs with high antimicrobial activity could be obtained $[8,9]$.

\section{Materials and Methods:-}

\section{Chemicals used:-}

The chemicals used like ethanol, agar, peptone, beef extract, Sodium chloride, Sodium hydroxide, Silver nitrate, etc were of analytical grade.

\section{Microbial strains used:-}

Following four microbial strains were used for the antimicrobial activity determination.

Gram + ve: Staphyloccous aureus (MTCC 9886) and Bacillus subtilis (MTCC 1789)

Gram - ve: Escherichia heranii(MTCC 9144) and Pseudomona aeruginosa (MTCC 10070)

[MTCC: Microbial type culture collection] that were obtained from the culture collection of the Microbial Type Culture Collection and Gene Bank (MTCC), Institute of Microbial Technology, Chandigarh, India and cultured and preserved in Institute of Pharmacy and Technology, Salipur microbiology laboratory.

\section{Preparation of Fruit extract:-}

The Fruits of Phyllanthus emblica were collected from the nearby village of Salipur, Odisha during early summer, washed in sterile water to remove any dirt or other unwanted objects. The fruit was cutted in to small pieces and seed was separated. The fruit pieces were shade dried and preserved. The dried biomass was powdered and accurately $10 \mathrm{gms}$ of powder biomass was taken in a conical flask added with double distilled water and boiled for 10 minutes and filtered to get the aqueous extract[10].

\section{Biosynthesis of silver nanoparticles:-}

In a typical synthesis for silver nanoparticles using aqueous fruit extract of Phyllanthus emblica, carefully $1 \mathrm{ml}$ of fruit extract was added to $50 \mathrm{ml}$ of $1 \mathrm{mM}$ aqueous Silver nitrate $\left(\mathrm{AgNO}_{3}{ }^{)}\right.$solution, in conical flasks of $250 \mathrm{ml} \mathrm{content}$ at room temperature[11].

\section{UV-Vis spectra analysis:}

The bioreduction of $\mathrm{Ag}+$ in aqueous solution was monitored by periodic sampling of aliquots $(0.2 \mathrm{ml})$ of the suspension, then diluting the samples with $2 \mathrm{ml}$ deionized water and subsequently measuring UV-vis spectra of the resulting diluents. UV-vis spectroscopy analyses of silver nanoparticles produced were carried out as a function of bioreduction time at room temperature on Shimadzu 1700 pharm spec UV spectrophotometers at a resolution of 1 $\mathrm{nm}[13]$.

\section{Size analysis:-}

Particle sizes of the prepared silver nanoparticles were determined by dynamic light scattering using a Malvern Zetasizer Nano-ZS. The nanoparticles suspended in purified water to a final concentration of $100 \mu \mathrm{g} / \mathrm{ml}$. The measurement was done in disposable polystyrene cuvettes at $25^{\circ} \mathrm{C}$ with a detection angle of $90^{\circ}$ and by adjusting the viscosity and refractive index that of water as $0.8872 \mathrm{cP}$ and 1.33 respectively. The particle size and the polydispersity index (PDI) of three batches of each sample were determined [14].

\section{SEM Analysis:-}

Scanning Electron Microscopic (SEM) Analysis was performed at a resolution: $0.19 \mathrm{~nm}$. Thin film of the sample were prepared on a carbon coated grid by dropping a very small amount of the sample on the grid, extra solution was removed using a blotting paper. Later on, film on the TEM grid was allowed to dry by placing it under a mercury lamp for 5 minutes for the characterization of size and shape of synthesized silver nanoparticles [15].

\section{FT-IR analysis:-}

To remove any free biomass residue or compound that is not the capping ligand of the nanoparticles, the residual solution of $100 \mathrm{ml}$ after reaction was centrifuged at $5000 \mathrm{rpm}$ for $10 \mathrm{~min}$ and the resulting suspension was redispersed in $10 \mathrm{ml}$ sterile distilled water. The centrifuging and redispersing process was repeated three times. 
Thereafter, the purified suspension was freeze dried to obtain dried powder. Finally, the dried nanoparticles were analyzed by Shimadzu 8400 S, FT-IR spectrophotometer. The sample is gently mixed with $100 \mathrm{mg}$ of micronized Potassium bromide powder and compressed into a disc shaped holder. For each spectrum a 256-scan interferogram was collected with a $4 \mathrm{~cm}^{-1}$ resolution in the mid-infrared region at room temperature[16].

\section{Determination of Minimum Inhibitory Concentration:-}

An agar dilution assay was used for determining the minimum inhibitory concentration (MIC) of the three extracts. This method was used as a modification of Parish and Davidson (1993). Tubes of $15 \mathrm{ml}$ molten agar were prepared and maintained at $50^{\circ} \mathrm{C}$. A single concentration of antimicrobial was added to the agar in each test tube to obtain a range of final concentrations of $10,20,30,40$ and $50 \mu \mathrm{g} / \mathrm{ml}$. After addition of the different concentration of extract to the agar, plates were poured and agar allowed solidifying. The test microorganisms were diluted in $0.1 \%(\mathrm{w} / \mathrm{v})$ peptone water to $10^{6} \mathrm{CFU} / \mathrm{ml}$. The test microorganism was then added to the plates and spreaded by a spreader. A control plate, without added antimicrobial, was prepared and inoculated to ensure adequate growth of the test microorganism. The plates were incubated at $37^{\circ} \mathrm{C}$ for overnight. The MIC was defined as the lowest concentration that completely inhibited growth up to $24 \mathrm{hr}[17]$.

\section{Antibacterial study:-}

For antibacterial activities, the samples were evaluated following a modified filter paper disc method. The samples were diluted to $50 \mu \mathrm{g} / \mathrm{ml}$ with sterilized distilled water. The sterile filter paper (Whatman filter paper) discs of $6 \mathrm{~mm}$ diameter were soaked with $50 \mu \mathrm{l}$ of the solution and placed on bacteria seeded plate $\left(10^{6} \mathrm{CFU} / \mathrm{ml}\right)$ of solid nutrient agar. For positive control, Cefexime in same concentration was used as similar manner. The plates were first incubated at $4^{\circ} \mathrm{C}$ for 12 hours to allow proper diffusion of the extract into the medium and then re-incubated at $37^{\circ} \mathrm{C}$ for 24 hours. After incubation period, the inhibition zone was observed and measured after marking at six different positions. The mean zone of inhibition and standard deviation was calculated by using SSP software [18].

\section{Result and discussion:-}

Biosynthesis of silver nanoparticles:-

Green synthesis of silver nanoparticles using $10^{-3} \mathrm{mM}$ Silver nitrate is shown in Figure 1. The fresh fruit extract of Phyllanthus emblica was yellowish-green in colour. However, after addition to Silver nitrate solution the colour was changed to dark brown.

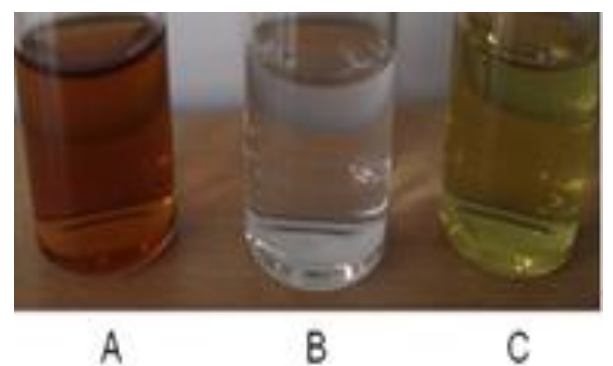

Figure 1:- Preparation of silver nanoparticles

[A: Silver nanoparticlesB: Only silver nitrate solutionC: Leave extract]

\section{UV-Vis Spectroscopy:-}

After addition of fresh fruit extract of Phyllanthus emblica to the aqueous solution of silver nanoparticles of different concentrations, the mixture showed a gradual change in color at room temperature with time from yellowish to wine-red and the colour intensified after 48 hours. The color developed was characteristic of the surface plasmon resonance (SPR) of silver nanoparticles. The control sets showed no change in color under the same experimental conditions. The reduction of silver ion to silver nanoparticle was reflected in spectral data obtained by using a UV-Vis spectrophotometer. It was shown an absorbance peak around $454 \mathrm{~nm}$ for all four samples (Figure 2), which is specific for silver nanoparticles. 


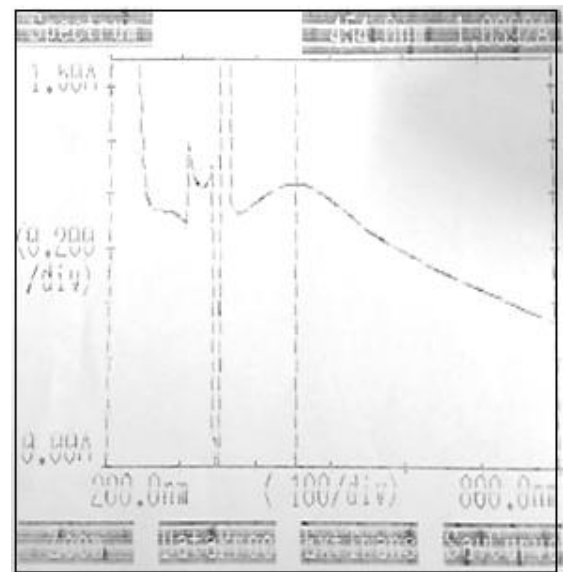

Figure 2: The absorbance spectrum of silver nanoparticles showing maximum absorbance at $454 \mathrm{~nm}$.

\section{Size analysis:-}

Light scattering technique is used here to determine the size distribution profile of nanoparticles present in the final solution after ultracentrifugation. The study revealed that the average particle size of $\mathrm{Ag}$ nanoparticles range within $20-60 \mathrm{~nm}$ with average size of approximately $36 \mathrm{~nm}$.

\section{SEM Analysis:-}

The grid for the SEM analysis of Ag-nanoparticles was prepared by placing a drop of the nanoparticles suspension on the carbon-coated copper grid and allowing the water to evaporate inside a vacuum dryer. Scanning under SEM revealed that the average mean size of silver nanoparticles was $20-50 \mathrm{~nm}$ and the tiny particles were seemed to be spherical in morphology as shown in the following images (Fig 3).

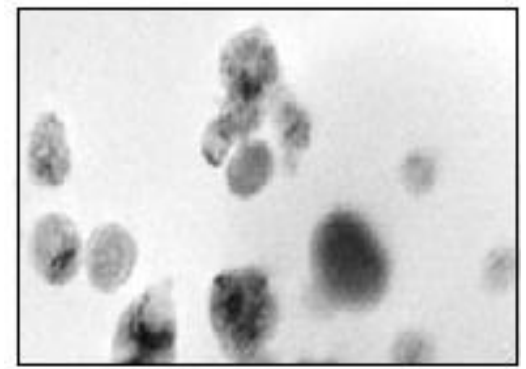

Figure 3: SEM images of silvernanoparticles.

\section{FT-IR analysis:-}

The FTIR analysis results of the Silvernanoparticles. The prominent peaks were observed at 1054, 1242, 1400, 1506 $\mathrm{cm}^{-1}$ and a peaks in the wave number $3375 \mathrm{~cm}^{-1}$, that is, amide region. That was indicating binding as well as stabilization takes place by free amide groups present in proteinaceous substance used for synthesis of silver nanoparticles. The peaks found at 1400 and $1506 \mathrm{~cm}^{-1}$ can be attributed to the $\mathrm{C}-\mathrm{C}$ in alkene rings and $\mathrm{C}=\mathrm{C}$ stretch of aromatic rings, respectively, whereas peaks at 1054 and $1242 \mathrm{~cm}^{-1}$ can be attributed to the ether linkages. It was confirmed from the observation that aliphatic amine, and aliphatic alkenes of alkaloids and terpenoids bound on the surface of silver nanoparticles. Depending on above observation, it can be assumed that the stabilization is achieved by the proteinaceous as well as aromatic compounds present in the extract.

\section{Determination of Minimum Inhibitory Concentration:-}

The minimum inhibitory concentration of silvernanoparticles against different microorganism was shown in Table 1.Staphylococcus aureus had shown MIC of $20 \mu \mathrm{g} / \mathrm{ml}$, Bacillus subtilis had shown MIC of $30 \mu \mathrm{g} / \mathrm{ml}$. Escherichia heranii and Pseudomonas aeruginosa had shown MIC of $40 \mu \mathrm{g} / \mathrm{ml}$. 
Table 1:- Minimum inhibitory concentration (MIC) of silvernanoparticles against different microorganism.

\begin{tabular}{|l|l|l|l|l|l|}
\hline \multirow{2}{*}{ Microorganisms } & \multicolumn{6}{l|}{ Concentration of silvernanoparticles } \\
\cline { 2 - 7 } & $10 \mu \mathrm{g} / \mathrm{ml}$ & $15 \mu \mathrm{g} / \mathrm{ml}$ & $20 \mu \mathrm{g} / \mathrm{ml}$ & $25 \mu \mathrm{g} / \mathrm{ml}$ & $30 \mu \mathrm{g} / \mathrm{ml}$ \\
\hline Staphylococusaureus & + & - & - & - & - \\
\hline Bacillus subtilis & + & + & - & - & - \\
\hline Escherichia heranii & + & + & + & - & - \\
\hline Pseudomonas aeruginosa & + & + & + & - & - \\
\hline
\end{tabular}

\section{Antimicrobial study:-}

Antibacterial activity of green synthesized silver nanoparticles against Gram negative Escherichia heranii, Pseudomonas aeruginosa and Gram positive Staphylococcus aureus, Bacillus subtilis bacteria at different concentrations showed that they revealed a strong dose-dependent antibacterial activity against the test microorganisms. It was seen that, as the concentration of green synthesized nanoparticles were increased, bacterial growth decreases in both the cases. The zone of inhibition of silver nanoparticles against the microorganisms Staphylococcus aureus, Bacillus subtilis, Escherichia heranii and Pseudomonas aeruginosa was shown in table 2. The results indicated that silvernanoparticles synthesized from fruit extract of Phyllanthus emblica showed antibacterial activity more in Gram positive than in Gram negative bacteria. As Gram negative bacteria were having high concentration of a lipid layer covering, that prevents penetration of silvernanoparticles inside the cell that may be a reason that gram negative microorganism were less sensitive towards silvernanoparticles as compared to gram positive microorganisms.

Table 2:- Antimicrobial activity of silvernanoparticles and antibiotic against tested microorganisms.

\begin{tabular}{|c|c|c|c|c|}
\hline \multirow{3}{*}{ Microorganisms } & \multicolumn{4}{|c|}{ Mean zone of inhibition in $\mathrm{cm}+/$-SD } \\
\hline & \multicolumn{3}{|c|}{ Silver nanoparticle } & Antibiotic \\
\hline & $500 \mu \mathrm{g} / \mathrm{ml}$ & $250 \mu \mathrm{g} / \mathrm{ml}$ & $100 \mu \mathrm{g} / \mathrm{ml}$ & $100 \mu \mathrm{g} / \mathrm{ml}$ \\
\hline Staphylococusaureus & $1.38+/-0.12$ & $0.97+/-.05$ & $0.68+/-.007$ & $1.32+/-0.07$ \\
\hline Bacillus subtilis & $1.25+/-.14$ & $0.85+/-0.1$ & $0.68+/-0.07$ & $1.17+/-0.05$ \\
\hline Escherichia heranii & $0.92+/-0.06$ & $0.77+/-0.05$ & $0.63+/-0.06$ & $0.8+/-0.06$ \\
\hline Pseudomonas aeruginosa & $0.88+/-0.07$ & $0.7+/-0.08$ & $0.63+/-0.05$ & $0.73+/-0.05$ \\
\hline
\end{tabular}

\section{Conclusion:-}

The rapid biological synthesis of silver nanoparticles using fruit extract of Phyllanthus emblica provides environmental friendly, simple and an efficient method for synthesis of benign nanoparticles. The synthesized nanoparticles were of spherical shape and the estimated sizes were in the range of 18-35 nm. The silver nanoparticles had shown antimicrobial activity against all the four tested microorganisms but more activity against gram positive microorganisms.

\section{References:-}

1. Bar H; Sahoo G; Sarkar P; Sankar PD;"Green synthesis of silvernanoparticles using latex of Jatropha curcas."Colliod surface A, 2009. 39(3), 134-139.

2. Atauro C M, De GaaetanoFD, MarottaA;Sol-gel processing of drug delivery materials and release kinetics.J Mater Sci Mater Med,2005; 16(3): 261-265.

3. Jiang HMS, Wong ACL, Denes FS;Plasma enhanced deposition of silver nanoparticles onto polymer and metal surfaces for the generation of antimicrobial characteristics.J ApplPolymSci,2004; 93: 1411-1422.

4. DuranNMP, AlvesOL, De SouzaGIH, EspositoE;Mechanistic aspects of biosynthesis of silver nanoparticles by several Fusariumoxysporum strains.J Nanobiotechnol, 2005; 3: 8-14.

5. ROB; Silver ions in the treatment of local infections. Met Based Drugs, 1999; 6: 297-300.

6. KlausTJR, Olsson E,GranqvistCG;Silverbased crystalline nanoparticles, microbially fabricated.Proc Natl AcadSci USA, 1999; 96: 13611-13614.

7. SinghalG,KasariyaK, SharmaAR,PalSinghR;Biosynthesis of silver nanoparticles using Ocimum sanctum (Tulsi) leaf extract and screening its antimicrobial activity.JNanopart Res, 2011; 13: 2981-2988.

8. Prusty AK;Preparation of silver nanoparticle by microorganism and its Application in pharmacy. Inter J Bio $A d v \operatorname{Res}, 2011 ; 02(01): 32-37$. 
9. Mukherjee AA, Mandal DS, Senapati S, Sainkar R, Khan MI, Parishcha R, Ajaykumar PV, Alam M, KumarR,SastryM;Fungus-mediated synthesis of silver nanoparticles and their immobilization in the mycelial matrix: a novel biological approach to nanoparticle synthesis. Nano Lett, 2001; 1: 515-519.

10. Prusty AK, GhoshT,Sahu S K, Anthelmintic,antimicrobial and antipyretic activity of various extracts of Clerodendrum infortunatumlinn. LeavesOri Pharm Exper Med, 2008; 8(4): 374-379.

11. Rajesh WR,NiranjanS K, Jaya R L, VijayD M,SahebraoB K; Extracellular synthesis of silver nanoparticles using dried leaves of pongamiapinnata (L) pierre. Nano-Micro.Lett,2010; 2: 106-113.

12. Shankar SS, Ahmad A, Sastry M; Geranium leaf assisted biosynthesis of silver nanoparticles.Biotechnology progress, 2003; 19:1627- 1631.

13. Schultz DA,Plasmon Resonant Particles for Biological Detection. Current Opinion Current Opinion Biotech,2003;14 (1): 13-22.

14. ShankarSS, RaiA,AnkamwarB, SinghA, AhmadA, and M. Sastry; Biological synthesis of triangular gold nanoprisms. Nature Materials, 2004; 3: 482-488.

15. WileyBJ,ImS H, LiZ Y, McLellanJ,SiekkinenA, XiaY; Maneuvering the surface plasmon resonance of silver nanostructures through shape-controlled synthesis. Journal of Physical Chemistry B,2006; 110(32): 1566615675 .

16. AhmadN, SharmaS; Green Synthesis of Silver Nanoparticles Using Extracts of Ananascomosus. Green and Sustainable Chemistry, 2012; 2: 141-147.

17. Kaviya S,SanthanalakshmiJ,ViswanathanB;Green Synthesis of Silver Nanoparticles Using Polyalthialongifolia Leaf Extract along with D-Sorbitol: Study of Antibacterial Activity.Journal of Nanotechnology, $2011: 1-5$.

18. Prusty AK, Samad L, Rout A, Patra A; In-Vitro Antibacterial Activity of Stereum Ostrea a Wood Decaying Macro Fungi. Journal of Microbiology Research and Reviews,2014; 2(2): 12-18. 